\title{
Construction and Practice of the Optimal Smooth Semi-Supervised Support Vector Machine
}

\author{
Xiaodan ZHANG \\ School of Mathematics and Physics, University of Science and Technology Beijing, Beijing 100083, \\ China \\ E-mail: bkdzxd@163.com
}

Ang LI

School of Mathematics and Physics, University of Science and Technology Beijing, Beijing 100083, China

E-mail: siwang744@gmail.com

Pan RAN

School of mechanical Engineering, Imperial College London, London SW59LS, UK

E-mail: r.pan13@imperial.ac.uk

\begin{abstract}
The standard semi-supervised support vector machine $\left(\mathrm{S}^{3} \mathrm{VM}\right)$ is an unconstrained optimization problem of non-convex and non-smooth, so many smooth methods are applied for smoothing $\mathrm{S}^{3} \mathrm{VM}$. In this paper, a new smooth semi-supervised support vector machine $\left(\mathrm{SS}^{3} \mathrm{VM}\right)$ model, which is based on the biquadratic spline function, is proposed. And, a hybrid Genetic Algorithm (GA)/ $\mathrm{SS}^{3} \mathrm{VM}$ approach is presented to optimize the parameters of the model. The numerical experiments are performed to test the efficiency of the model. Experimental results show that generally our optimal $\mathrm{SS}^{3} \mathrm{VM}$ model outperforms other optimal $\mathrm{SS}^{3} \mathrm{VM}$ models mentioned in this paper.
\end{abstract}

Keywords semi-supervised support vector machine; classification; genetic algorithm; smooth; spline function

\section{Introduction}

Support vector machines (SVMs) were introduced by Vapnik ${ }^{[1]}$ in the early 1990s. As an effective method of data mining, SVMs are widely applied in many fields ranging from text categorization $^{[2]}$, image retrieval ${ }^{[3]}$, face recognition ${ }^{[4]}$, intelligent goal-driven ${ }^{[5]}$, information security $^{[6]}$ to credit risk evaluation ${ }^{[7]}$, bankruptcy prediction ${ }^{[8]}$, time series forecasting ${ }^{[9]}$, etc. The standard SVM is based on supervised learning. In this result, a large number of labeled samples are required to ensure the preferable classification accuracy. But manual labelling is often a slow, expensive, and error prone process. By contrast, the semi-supervised support vector machines $\left(\mathrm{S}^{3} \mathrm{VMs}\right)^{[10-12]}$ can take advantage of labeled samples and unlabeled samples simultaneously, and then reduce the labelling cost significantly. Hence, $\mathrm{S}^{3} \mathrm{VM}$ has widely attracted attention of some researchers ${ }^{[13-15]}$. Since the unconstrained optimization problem of 
$\mathrm{S}^{3} \mathrm{VM}$ is non-convex and non-smooth, most of the fast algorithms cannot be used to solve the $\mathrm{S}^{3} \mathrm{VMs}$ problem. With this regard, many famous researchers were involved in the development of smooth $\mathrm{S}^{3} \mathrm{VM}\left(\mathrm{SS}^{3} \mathrm{VM}\right)$ in recent years ${ }^{[15-18]}$. The $\mathrm{SS}^{3} \mathrm{VM}$ models, smoothed by Gaussian function $^{[12]}$, polynomial functions ${ }^{[19]}$, and the cubic spline function ${ }^{[20]}$, have been proposed successively. However, the influences of all parameters of $\mathrm{SS}^{3} \mathrm{VM}$ on the classification results have not been deeply studied. In fact, the effects of parameters on the performance of $\mathrm{SS}^{3} \mathrm{VM}$ cannot be ignored. Therefore, selecting the appropriate parameters of $\mathrm{SS}^{3} \mathrm{VM}$ is crucial.

In this paper, we firstly explore the smoothing method and propose a new $\mathrm{SS}^{3} \mathrm{VM}$ model based on a biquadratic spline function. Secondly, we focus on the optimization of parameters of the $\mathrm{SS}^{3} \mathrm{VM}$ models. A hybrid approach combined with the Genetic Algorithm (GA) ${ }^{[21]}$ and $\mathrm{SS}^{3} \mathrm{VM}$ is introduced. Finally, we evaluate the new model through numerical experiments. Four dataset are used to test the efficiency of the new model. Moreover, the effect of the number of labeled samples on the classification accuracy of the new model is analyzed.

\section{The $\mathrm{SS}^{3} \mathrm{VM}$ Model}

We consider the two-class classification problem. The dataset consist of $m$ labeled samples $\left\{\left(\boldsymbol{x}_{i}, y_{i}\right)\right\}_{i=1}^{m}$ and $l$ unlabeled samples $\left\{\overline{\boldsymbol{x}}_{i}\right\}_{i=1}^{l}$, where $\boldsymbol{x}_{i}, \overline{\boldsymbol{x}}_{i} \in \boldsymbol{R}^{n}, y_{i} \in\{1,-1\} . \boldsymbol{x}_{i}(i=$ $1,2, \cdots, m)$ and $\overline{\boldsymbol{x}}_{i}(i=1,2, \cdots, l)$ are represented by an $m \times n$ matrix $\boldsymbol{A}_{m \times n}$ and $\boldsymbol{B}_{l \times n}$, respectively. The result of classification for labeled samples is determined by an $m \times m$ diagonal matrix $\boldsymbol{D}$ with 1 or -1 along its diagonal. Precisely, a standard unconstrained optimization model of $\mathrm{S}^{3} \mathrm{VM}$ is given as follows ${ }^{[22]}$ :

$$
\min _{\boldsymbol{\omega}, b} \frac{1}{2}\|\boldsymbol{\omega}\|_{2}^{2}+c \boldsymbol{e}_{1}^{\mathrm{T}} \Lambda\left(\boldsymbol{D}\left(\boldsymbol{A} \boldsymbol{\omega}+\boldsymbol{e}_{1} b\right)\right)+c^{*} \boldsymbol{e}_{2}^{\mathrm{T}} \Lambda\left(\left|\left(\boldsymbol{B} \boldsymbol{\omega}+\boldsymbol{e}_{2} b\right)\right|\right)
$$

Here, $\boldsymbol{\omega}$ is the normal vector to the bounding plane, $b$ is a bias value, $\boldsymbol{e}_{i}(i=1,2)$ is a column vector of 1's, with $\boldsymbol{e}_{1} \in \boldsymbol{R}^{m}, \boldsymbol{e}_{2} \in \boldsymbol{R}^{l} . c$ and $c^{*}$ are the penalty parameters. $\Lambda(t)=\max (0,1-t)$ is called the hinge loss function and $\Lambda(|t|)=\max (0,1-|t|)$ is called the symmetric hinge loss function $^{[6]}$. If $\boldsymbol{u}=\left(u_{1}, u_{2}, \cdots, u_{n}\right) \in \boldsymbol{R}^{n}$, then $\Lambda(\boldsymbol{u})=\left(\Lambda\left(u_{1}\right), \Lambda\left(u_{2}\right), \cdots, \Lambda\left(u_{n}\right)\right)^{\mathrm{T}}$. Nevertheless, the $\Lambda(t)$ and $\Lambda(|t|)$ are not differentiable. As a result, the objective function in the Model (1) is non-convex and non-smooth to which precludes the applications of many fast optimization methods such as BFGS algorithm ${ }^{[23,25]}$. So we modify the Model (1) as below:

$$
\min _{\boldsymbol{\omega}, b} \frac{1}{2}\|\boldsymbol{\omega}\|_{2}^{2}+\frac{c}{2}\left\|\Lambda\left(\boldsymbol{D}\left(\boldsymbol{A} \boldsymbol{\omega}+\boldsymbol{e}_{1} b\right)\right)\right\|_{2}^{2}+\frac{c^{*}}{2}\left\|f\left(\boldsymbol{B} \boldsymbol{\omega}+\boldsymbol{e}_{2} b, r\right)\right\|_{2}^{2}
$$

Here, $f(\boldsymbol{x}, r)$ is an arbitrary smooth function at origin, which is used to approximate the symmetric hinge loss function and $r$ is the relevant parameter. The 1-norm being substituted by 2 -norm avoid the non-smoothness of the hinge loss function $\Lambda(\boldsymbol{x})$ at +1 and the symmetric hinge loss function at +1 and -1 . In next section, a biquadratic spline function is constructed to replace the $f(\boldsymbol{x}, r)$ in Model (2). 


\section{The $\mathrm{SS}^{3} \mathrm{VM}$ Model Based on the Biquadratic Spline Function}

\subsection{Construction of Biquadratic Spline Function}

Definition 1 For $k>1, y_{1}>0, m, n \in Z_{+}$, let $x_{0}=-\frac{1}{k}, x_{1}=0, x_{2}=\frac{1}{k}$ be a set of nodes. The function $s(x, k)$ is defined as follows:

$$
s(x, k)= \begin{cases}s_{0}(x, k), & -\frac{1}{k} \leq x<0 \\ s_{1}(x, k), & 0 \leq x \leq \frac{1}{k} \\ \Lambda(|x|), & |x|>\frac{1}{k}\end{cases}
$$

Here $s_{0}(x, k)$ and $s_{1}(x, k)$ are the $n$-times polynomials. If $s(x, k)$ satisfies the following conditions:

(a) $s^{(d)}\left(x_{0}, k\right)=0, d=2,3, \cdots, m ; k>0, s^{\prime}\left(x_{0}, k\right)=1, s\left(x_{0}, k\right)=1-\frac{1}{k} ;$

(b) $s^{(d)}\left(x_{2}, k\right)=0, d=2,3, \cdots, m ; k>0, s^{\prime}\left(x_{2}, k\right)=-1, s\left(x_{2}, k\right)=1-\frac{1}{k}$;

(c) $s_{0}{ }^{(d)}\left(x_{1}-0, k\right)=s_{1}{ }^{(d)}\left(x_{1}+0, k\right), d=1,2, \cdots, m, s_{0}\left(x_{1}+0, k\right)=s_{1}\left(x_{1}-0, k\right)=y_{1}$.

Then it is called the $n$-times spline function with the m-orders smooth condition at origin which approximating $\Lambda(|x|)$.

Theorem 1 Let $k>1$ and $x_{0}=-\frac{1}{k}, x_{1}=0, x_{2}=\frac{1}{k}$ be a set of nodes. Then there exists a unique biquadratic spline $s(x, k)$ with the second derivative at origin approximating $\Lambda(|x|)$. The function must have the following expression:

$$
s(x, k)= \begin{cases}\frac{1}{8} k^{3} x^{4}-\frac{3}{4} k x^{2}-\frac{3}{8 k}+1, & -\frac{1}{k} \leq x \leq \frac{1}{k} \\ \Lambda(|x|), & |x|>\frac{1}{k}\end{cases}
$$

Proof Let $s(x, k)$ be a quartic spline function with the second derivative at origin, which satisfies the conditions in Definition 1. We derive the equation for $s(x, k)$ on $\left[-\frac{1}{k}, \frac{1}{k}\right]$. Set $s^{(3)}\left(x_{i}, k\right)=M_{i}(i=0,1,2) . \forall x \in\left[-\frac{1}{k}, 0\right]$, it has $s(x, k)=s_{0}(x, k)$. Since $s_{0}(x, k)$ is a polynomial of degree four on $\left[-\frac{1}{k}, 0\right], s_{0}{ }^{(3)}(x, k)$ is a linear function. The expression of $s_{0}{ }^{(3)}(x, k)$ can be given by

$$
s_{0}^{(3)}(x, k)=M_{0} \frac{\left(x_{1}-x\right)}{h_{0}}+M_{1} \frac{\left(x-x_{0}\right)}{h_{0}}=-M_{0} k x+M_{1} k\left(x-x_{0}\right)
$$

The above equation is integrated successively 3 times, we obtain:

$$
s_{0}(x, k)+\frac{a_{1}}{2} x^{2}+a_{2} x+a_{3}=-\frac{M_{0} k x^{4}}{4 !}+\frac{M_{1} k}{4 !}\left(x-x_{0}\right)^{4}
$$

where $a_{1}, a_{2}$, and $a_{3}$ are constants of the integration. According to condition (a) in Definition 1, we can determine: $a_{1}=-\frac{M_{0}}{2 k}, a_{2}=-\frac{M_{0}}{3 k^{2}}-1, a_{3}=-\frac{M_{0}}{8 k^{3}}-1$.

Similarly, on $\left(0, \frac{1}{k}\right]$, we have

$$
s_{1}(x, k)+\frac{b_{1}}{2} x^{2}+b_{2} x+b_{3}=\frac{M_{2} k x^{4}}{4 !}-\frac{M_{1} k}{4 !}\left(x_{2}-x\right)^{4}
$$

where $b_{1}, b_{2}$, and $b_{3}$ are constants of the integration. Based on condition (b) in Definition 1, it follows that $b_{1}=\frac{M_{2}}{2 k}, b_{2}=-\frac{M_{2}}{3 k^{2}}+1, b_{3}=\frac{M_{2}}{8 k^{3}}-1$. 
In this way, we show that $s(x, k)$ is piecewise polynomial of degree 4 with parameters $M_{0}$, $M_{1}$, and $M_{2}$ on $\left[-\frac{1}{k}, \frac{1}{k}\right]$. Furthermore, applying condition (c) in Definition 1, we obtain the matrix equation:

$$
\left[\begin{array}{ccc}
3 & 2 & 3 \\
\frac{1}{3 k^{2}} & 0 & -\frac{1}{3 k^{2}} \\
1 & 2 & 1
\end{array}\right] \cdot\left[\begin{array}{c}
M_{0} \\
M_{1} \\
M_{2}
\end{array}\right]=\left[\begin{array}{c}
0 \\
-2 \\
0
\end{array}\right]
$$

Because the coefficient matrix of Equation (7) is nonsingular, we can get the unique solution $M_{0}=-3 k^{2}, M_{1}=0$, and $M_{2}=3 k^{2}$. Finally, the biquadratic spline function $s(x, k)$ with the second derivative at origin approximating $\Lambda(|x|)$ is obtained as Equation (4).

Theorem 2 Let $\Lambda(|x|)$ be the symmetric hinge loss function and $s(x, k)$ be the general biquadratic spline function given in Model (4). Then, for $x \in \boldsymbol{R}$ and $k>1$, we have:

(a) $0 \leq s(x, k) \leq \Lambda(|x|)$;

(b) $0 \leq \Lambda^{2}(|x|)-s^{2}(x, k) \leq \frac{3}{8 k}\left(2-\frac{3}{8 k}\right)$.

Proof If $x \in\left(\frac{1}{k},+\infty\right) \cup\left(-\infty,-\frac{1}{k}\right)$, both the inequalities (a) and (b) are true, since $s(x, k)=\Lambda(|x|)$. It remains to show that the inequalities are true on $x \in\left[-\frac{1}{k}, \frac{1}{k}\right]$.

(a). If $x \in\left[-\frac{1}{k}, 0\right]$, then $s(x, k)=s_{0}(x, k)=\frac{1}{8} k^{3} x^{4}-\frac{3}{4} k x^{2}-\frac{3}{8 k}+1$. Thus $s_{0}^{(2)}(x, k)=$ $\frac{3}{2} k^{3} x^{2}-\frac{3}{2} k \leq 0 \Rightarrow \min _{x} s_{0}^{\prime}(x, k)=s_{0}^{\prime}(0, k)=0$. Hence, $s(x, k)$ is an increasing function. It follows that

$$
1-\frac{3}{8 k}=s_{0}(x, k) \geq s_{0}\left(-\frac{1}{k}, k\right)=1-\frac{1}{k} \geq 0
$$

In addition, let $\lambda(x, k)=\Lambda(|x|)-s_{0}(x, k)=x-\frac{1}{8} k^{3} x^{4}+\frac{3}{4} k x^{2}+\frac{3}{8 k}$. Then

$$
\lambda^{(2)}(x, k)=-\frac{3}{2} k^{3} x^{2}+\frac{3}{2} k \geq 0 \Rightarrow \lambda^{\prime}(k, x) \geq 0
$$

Therefore, $\lambda(x, k)$ is also an increasing function on $\left[-\frac{1}{k}, 0\right] \cdot \min _{x} \lambda(x, k)=\lambda\left(-\frac{1}{k}, k\right)=0$ is obtained. So $0 \leq s_{0}(x, k) \leq \Lambda(|x|)$, when $x \in\left[-\frac{1}{k}, 0\right]$.

If $x \in\left(0, \frac{1}{k}\right], s(x, k)=s_{1}(x, k)$. Similar to the first part, the inequality (a) can also be obtained.

(b). If $x \in\left[-\frac{1}{k}, 0\right]$, since $\lambda(x, k)$ is an increasing, the maximum $\frac{3}{8 k}$ can be obtained at the point $x=0$. Hence,

$$
0 \leq \Lambda(|x|)-s_{0}(x, k) \leq \frac{3}{8 k}
$$

In addition, $\Lambda(|x|)+s_{0}(x, k)=\lambda(x, k)+2 s_{0}(x, k)$. Inequalities (8) and (10) imply that: $\Lambda(|x|)+s_{0}(x, k) \leq 2-\frac{3}{8 k}$.

Thus, $0 \leq \Lambda^{2}(|x|)-s_{0}^{2}(x, k) \leq \frac{3}{8 k}\left(2-\frac{3}{8 k}\right)$.

Similarly, if $x \in\left(0, \frac{1}{k}\right]$, we have $0 \leq \Lambda^{2}(|x|)-s^{2}(x, k) \leq \frac{3}{8 k}\left(2-\frac{3}{8 k}\right)$ as well.

In order to show the approximating accuracy of different smooth functions, the comparison diagram of smooth performance is shown in Figure 1. 


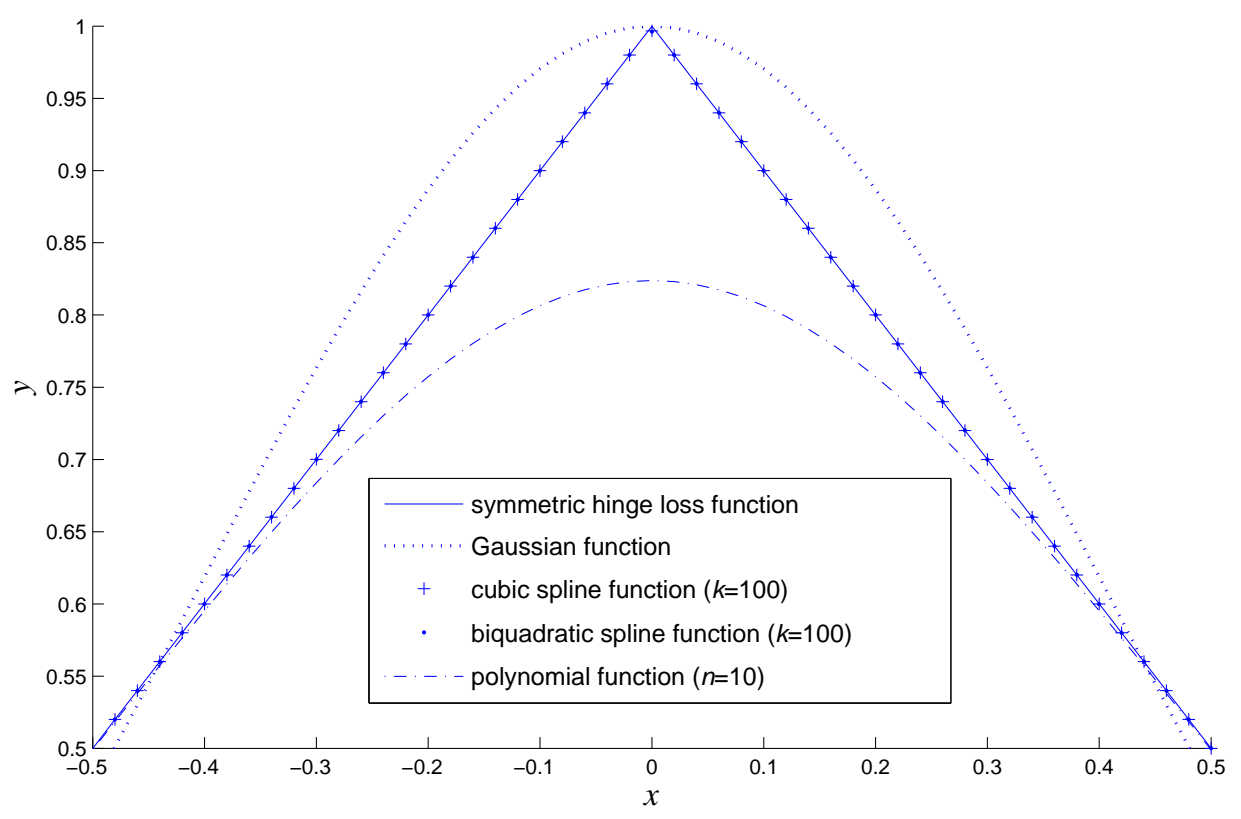

Figure 1 Different smooth functions approximate $\Lambda(|x|)$

As shown in Figure 1, the biquadratic spline function is closer to the symmetric hinge loss function than both the Gaussian function and the polynomial function. Though the approximate accuracy of the biquadratic spline function is slightly lower than that of the cubic spline function, the biquadratic spline function has more simple expression.

\subsection{The Smooth $\mathrm{S}^{3}$ VM Model Based on the Biquadratic Spline Function}

$f(x)$ in Model (2) is replaced by the $s(x, k)$ in the Equation (4). We obtain the following biquadratic spline smooth semi-support vector machine model:

$$
\min _{\boldsymbol{\omega}, b} \frac{1}{2}\|\boldsymbol{\omega}\|_{2}^{2}+\frac{c}{2}\left\|\Lambda\left(\boldsymbol{D}\left(\boldsymbol{A} \boldsymbol{\omega}+\boldsymbol{e}_{1} b\right)\right)\right\|_{2}^{2}+\frac{c^{*}}{2}\left\|s\left(\boldsymbol{B} \boldsymbol{\omega}+\boldsymbol{e}_{2} b, k\right)\right\|_{2}^{2}
$$

where $k$ is the smooth parameter.

\section{The Optimal $\mathrm{SS}^{3} \mathrm{VM}$ Model Based on GA}

One of the big problems in $\mathrm{SS}^{3} \mathrm{VM}$ is the selection of the values of parameters that will allow good performance. But, it is not known beforehand what values are the best for the model. In order to select the suitable parameters of the $\mathrm{SS}^{3} \mathrm{VM}$, the GA is applied ${ }^{[21,26-28]}$. GA is an artificial intelligence procedure based on the theory of natural selection and evolution. Unlike the conventional optimization methods, it has the advantages consisting of parallel search, solving complex problems, and large search space.

The $\mathrm{SS}^{3} \mathrm{VM}$ training algorithm and GA are combined to optimize the parameters of $\mathrm{SS}^{3} \mathrm{VM}$. For ease of notation, we named this hybrid method as GA/SS ${ }^{3} \mathrm{VM}$ method. Figure 2 shows the overall procedure of the $\mathrm{GA} / \mathrm{SS}^{3} \mathrm{VM}$ method.

The detailed steps of GA/SS ${ }^{3} \mathrm{VM}$ method are given as below: 
Step 1 Define the string (or chromosome).

According to the Model (2), the parameters $c, c^{*}$, and $r$ need to be optimized. So, the individual is defined as $\boldsymbol{x}=\left(c, c^{*}, r\right)$. The chromosome of $\boldsymbol{x}$ is encoded as a $\boldsymbol{l}$-bit string which consists of $l_{1}$ bits standing for $c, l_{2}$ bits standing for $c^{*}$, and $l_{1}$ bits standing for $r$. Here, $l=\left(l_{1}+l_{2}+l_{3}\right)$.

Step 2 Determine the fitness function.

The fitness of an individual of population is based on the performance of SVM. So, the prediction accuracy of $\mathrm{SS}^{3} \mathrm{VM}$ is taken as the fitness function $F(\boldsymbol{x})$.

Step 3 Initialization.

Step 3.1 Define the size of population $N$, probability of crossover $P_{c}$, and probability of mutation $P_{m}$.

Step 3.2 Generate an initial population consisted of $N$ l-bit strings described in Step 1 randomly: $P(L)=\left\{\boldsymbol{x}_{j}(L)=\left(c_{j}, c_{j}^{*}, r_{j}\right)_{L}, j=1,2, \cdots, N\right\}$, where $L$ is the number of the current generation population. In initial generation, $L=0$.

Step 4 Decode the $j$ th string to obtain the corresponding individual $\boldsymbol{x}_{j}(L)=\left(c_{j}, c_{j}^{*}, r_{j}\right)_{L}$.

Step 5 Apply $\boldsymbol{x}_{j}(L)$ to the $\mathrm{SS}^{3} \mathrm{VM}$ model to compute the Fitness $F\left(\boldsymbol{x}_{j}(L)\right)$.

Step 6 Evolution;

Step 6.1 Find the worst fitness $F_{\text {min }}^{L}$, the best fitness $F_{\max }^{L}$, and the corresponding $\boldsymbol{x}_{\min }^{L}, \boldsymbol{x}_{\min }^{L}$ in the $L$ th generation population.

Step 6.2 Replace $\boldsymbol{x}_{\min }^{L}$ with $\boldsymbol{x}_{\min }^{L}$.

Step 7 Calculate the fitness of the $L$ th generation population: $T_{L}=\sum_{j=1}^{N} F\left(\boldsymbol{x}_{j}(L)\right)$.

Step 8 Reproduction.

Step 8.1 Compute cumulative probabilities $q_{j}=\sum_{i=1}^{j} p_{i}(j=1,2, \cdots, N)$, where $p_{i}=$ $\frac{F\left(\boldsymbol{x}_{i}(L)\right)}{T_{L}}$.

Step 8.2 Generate $N$ random numbers $r_{1 i}, i=1,2, \cdots, N$ in $[0,1]$. For each $r_{1 i}$, If $q_{j-1}<r_{1 i} \leq q_{j}$, then select the $j$ th string, otherwise, select the first string such that $r_{1 i} \leq q_{1}$.

Step 9 Generate offspring population $P(L+1)$ by performing crossover and mutation.

Step 9.1 Crossover: For two parent individuals, a random number $r_{2}$ in $[0,1]$ is generated. If $r_{2}<P_{c}$, choose a random crossover point and exchange the genetic code of the two parent individuals on this point to obtain two new child individuals.

Step 9.2 Mutation: Generate a random number $r_{3}$ in $[0,1]$ and select a bit randomly. If $r_{3}<P_{m}$, then operate mutation for the bit. 
Step 10 If the terminal condition is satisfied, output the best individual $\boldsymbol{x}_{\max }^{(L+1)}$. Otherwise, do Steps $4 \sim 10$. Terminal condition: The maximum number of generations $L_{\max }$ is reached or the optimum individual does not improve during successive generations.

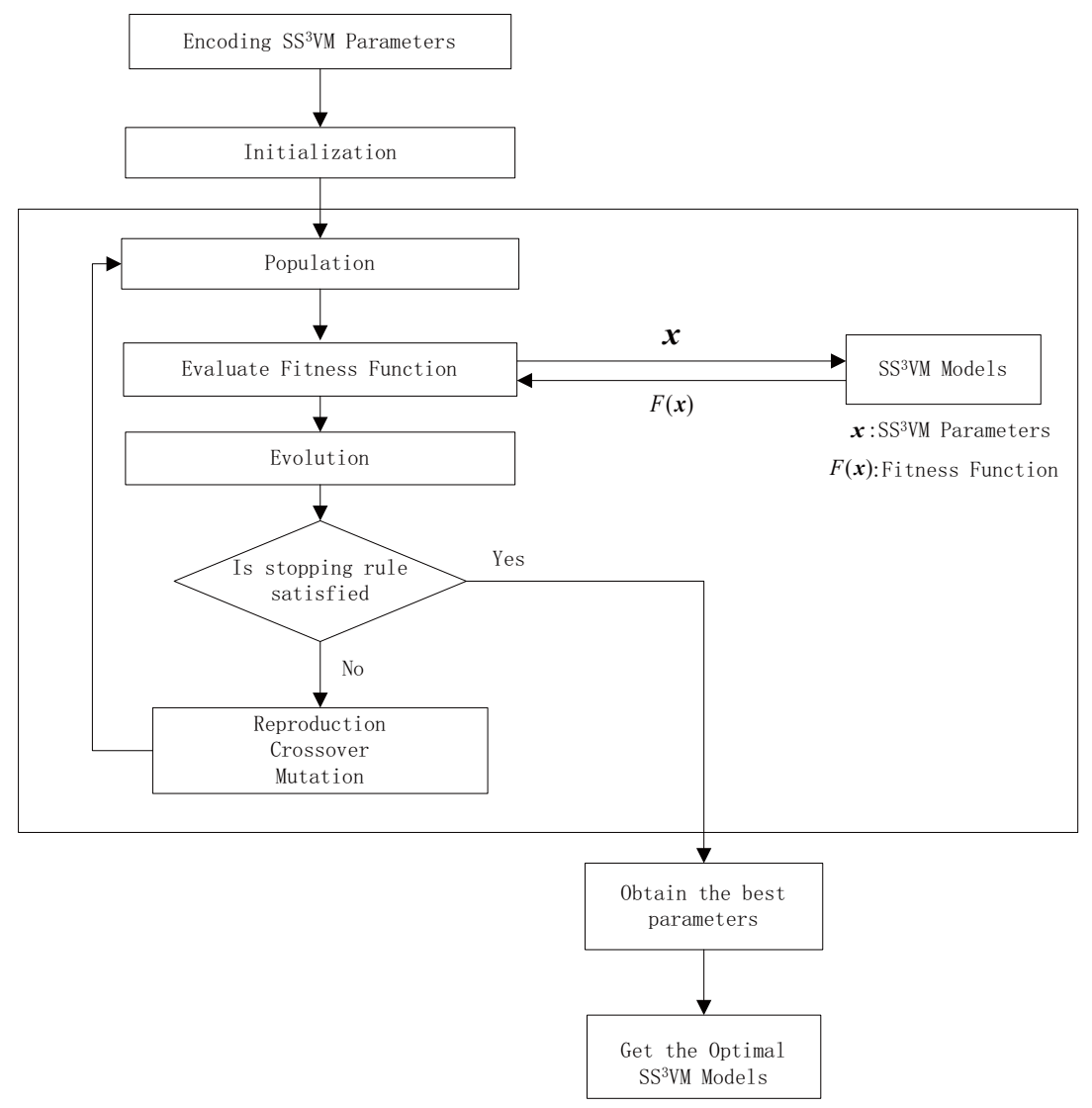

Figure 2 Overall procedure of $\mathrm{GA} / \mathrm{SS}^{3} \mathrm{VM}$ method

\section{Data Preparation}

\subsection{Datasets}

In this section, four datasets are used to test the hybrid GA/ $\mathrm{SS}^{3} \mathrm{VM}$ method and our new $\mathrm{SS}^{3}$ VM model. All the datasets are obtained from UCI Machine Learning Library (http://archive. ics.uci.edu/ml/). The datasets are named as Heart, QSAR, Wine, and Wilt, respectively. In Heart dataset, the data includes features of the heart disease patients. Heart disease conditions are divided into two categories: presence and absence. The QSAR dataset is used to develop QSAR (Quantitative Structure Activity Relationships) models and study the relationships between chemical structure and biodegradation of molecules. The purpose is to discriminate molecules being ready biodegradable molecules or not. The Wine dataset is related to the white wine quality. The dataset includes objective data and sensory data. The objective data consists of 11 physicochemical attributes such as fixed acidity, volatile acidity and etc. The sensory data includes the median scores of wine quality from 0 (very bad) to 10 (very excellent) graded by experts. In our experiment, if the quality score is bigger than 5 , the wine quality 
is considered as excellent, otherwise, the wine quality is considered as poor. The Wilt dataset involves detecting diseased trees in Quickbird imagery. There are samples for the "diseased trees" class and many for "other land cover" class. For the above four datasets, each sample has been marked. The detailed information of the above datasets is shown in Table 1.

Table 1 Information of the four datasets

\begin{tabular}{cccl}
\hline Data sets & Classes & Samples & Attributes \\
\hline Heart & 2 & 270 & 13 (age, sex, cp, etc) \\
QSAR & 2 & 1056 & 42 (nHM, thenCp, etc) \\
Wine & 2 & 4898 & 12 (fixed acidity, citric acid, etc) \\
Wilt & 2 & 4339 & 5 (GLCM_Pan, Mean_G, etc) \\
\hline
\end{tabular}

\subsection{Data Pre-Processing}

\subsubsection{Data Reduction}

The above datasets, especially QSAR, contain a large number of attributes. If all the ratios are used as inputs of $\mathrm{SS}^{3} \mathrm{VM}$, it would result in redundancy and low efficiency. So, the principal component analysis (PCA) is applied to avoid these problems. The $\mathrm{PCA}^{[29-32]}$ can be used for reducing complexity of input variables and it is intended to have a better interpretation of variables. Let $X_{i}$ be the original variable and $\boldsymbol{R}$ be a variance-covariance matrix. Then, the principal components can be expressed as follows:

$$
Y_{i}=\alpha_{i} \cdot X=\alpha_{i 1} X_{1}+\alpha_{i 2} X_{2}+\cdots+\alpha_{i p} X_{p}
$$

For the above four data sets, the numbers of their original attributes are 13, 42, 12, and 5 , respectively. The number of attributes is integrated into 10, 15, 8, and 4 by PCA. In this result, the useless information redundancy and the computational complexity are reduced.

\subsubsection{Data Normalization}

In order to avoid the numerical difficulties caused by different numeric ranges of variables, the selected input variable is normalized by $\boldsymbol{x}_{\text {scoled }}=2\left(\boldsymbol{x}-x_{\min }\right) /\left(x_{\max }-x_{\min }\right)-1$. Here, $\boldsymbol{x}$ is the original variable, $x_{\max }, x_{\min }$ is the maximum, the minimum of original variable, respectively.

\section{$6 \quad$ Numerical Experiments}

\subsection{Experimental Design}

In order to test GA/SS ${ }^{3} \mathrm{VM}$ method and the new Model (11), the three other smooth $\mathrm{SS}^{3} \mathrm{VM}$ models are applied in our experiments. For ease of notation, these $\mathrm{SS}^{3} \mathrm{VM}$ models are named as follows:

(i) The $\mathrm{SS}^{3} \mathrm{VM}$ smoothed by Gaussian function is named as $\mathrm{GSS}^{3} \mathrm{VM}$;

(ii) The $\mathrm{SS}^{3} \mathrm{VM}$ smoothed by a polynomial function is named as $\mathrm{PSS}^{3} \mathrm{VM}$;

(iii) The $\mathrm{SS}^{3} \mathrm{VM}$ smoothed by the cubic spline function is named as $3 \mathrm{SS}^{3} \mathrm{VM}$;

(iv) The $\mathrm{SS}^{3} \mathrm{VM}$ Model (11) is named as $4 \mathrm{SS}^{3} \mathrm{VM}$.

The experiments are divided into three parts as follows: 
(i) Compare performance of four $\mathrm{SS}^{3} \mathrm{VM}$ models optimized by $\mathrm{GA} / \mathrm{SS}^{3} \mathrm{VM}$ method with that of the original ones.

(ii) Compare performance of the optimal $4 \mathrm{SS}^{3} \mathrm{VM}$ model with that of other three optimal $\mathrm{SS}^{3} \mathrm{VM}$ models.

(iii) Evaluate the classification accuracy sensitivity of the optimal $4 \mathrm{SS}^{3} \mathrm{VM}$ model to the different proportion of labeled samples.

Here, the $\mathrm{SS}^{3} \mathrm{VM}$ models are solved by BFGS algorithm and the performance of models is evaluated by classification accuracy and CPUtime.

Since the samples of above four datasets are all labeled. For performing semi-supervised experiments, the unlabeled samples are simulated by dropping labels from some labeled samples. Each dataset is randomly separated into two portions: training set and testing set. The training set is used to train the models and select the optimal parameters, and the testing set is applied to evaluate the performance of the models. The ratios of two portions are about 0.7 and 0.3 . In the first two part of the experiment, for either training set or testing set of each dataset, the proportion of the labeled samples is set as $1 / 5$, i.e., the $20 \%$ samples are chosen as labeled and the rest are processed as unlabeled. And, for each training set, the GA/ $\mathrm{SS}^{3} \mathrm{VM}$ method is used to optimize parameters of $\mathrm{SS}^{3} \mathrm{VM}$ model. For the four models: GSS ${ }^{3} \mathrm{VM}, \mathrm{PSS}^{3} \mathrm{VM}$, $3 \mathrm{SS}^{3} \mathrm{VM}$, and $4 \mathrm{SS}^{3} \mathrm{VM}$, the parameters to be optimized, are $\left(c, c^{*}\right),\left(c, c^{*}, n\right),\left(c, c^{*}, k\right)$, and $\left(c, c^{*}, k\right)$ respectively. Here, $k$ is the smooth parameter and $n$ is the degree of the polynomial function. Otherwise, an assumption is applied that, during the optimization process, the labels found for the unlabeled samples are right. So, $c=c^{*}$ is set. The parameters of GA used to optimize these models are shown in Table 2.

Table 2 GA parameters for optimizing models

\begin{tabular}{ll}
\hline Parameters & Value \\
\hline Size of population $(N)$ & 40 \\
Maximum Number of generations $(\max I)$ & 70 \\
Length of chromosome of $c\left(c^{*}=c\right)$ & 5 \\
Length of chromosome of $k$ & 12 \\
Length of chromosome of $n$ & 3 \\
Crossover rate $\left(P_{c}\right)$ & 0.7 \\
Mutation rate $\left(P_{m}\right)$ & 0.01 \\
\hline
\end{tabular}

In part three of the experiment, the sensitivity of the optimal $4 \mathrm{SS}^{3} \mathrm{VM}$ model to the proportion of labeled samples is tested. For each the dataset, we change the proportion of labeled samples to observe the volatility of classification accuracy of the optimal $4 \mathrm{SS}^{3} \mathrm{VM}$ model. Here, we arbitrarily select $20 \%, 30 \%, 40 \%$, and $50 \%$ as the proportion of the labeled samples.

\subsection{Experimental Results and Comparisons}

In this section, the results that corresponding to the three parts of the experiments are obtained and analysed. Figure 3 shows the evolution process of the optimal parameters selection on Heart dataset. As it is shown in Figure 3, for four $\mathrm{SS}^{3} \mathrm{VM}$ models, the stop criterion of GA process is satisfied at about 10 th or 15 th generation $(L=10$ or $L=15)$, i.e., the 
optimal parameters of four $\mathrm{SS}^{3} \mathrm{VM}$ models are obtained at about 10th or 15th generation. The procedures of the parameters selection for QSAR, Wine, and Wilt datasets are similar to that of the Heart dataset. It is emphasized that the selection procedures are distinct for different training sets. Moreover, the process is likely not constant for same training sets because of the stochastic of GA. Table 3 summarizes the optimal parameters for the different $\mathrm{SS}^{3} \mathrm{VM}$ models on the above four datasets. The training accuracy, testing accuracy, and training time are also shown in Table 3 . As it can be observed, comparing with other models, the $4 \mathrm{SS}^{3} \mathrm{VM}$ model achieves the preferable training accuracy and testing accuracy on four datasets with less CPUtime.

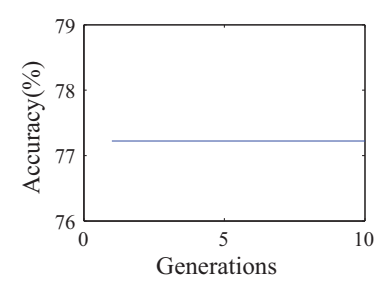

(a) $\mathrm{GSS}^{3} \mathrm{VM}$

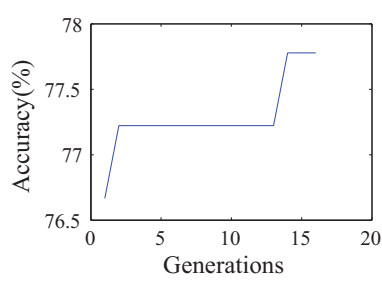

(c) $3 \mathrm{SS}^{3} \mathrm{VM}$

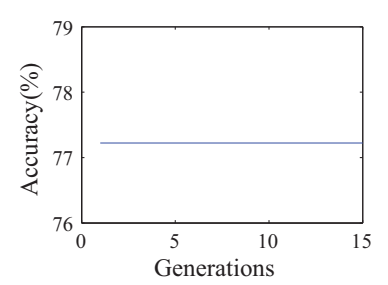

(b) $\mathrm{PSS}^{3} \mathrm{VM}$

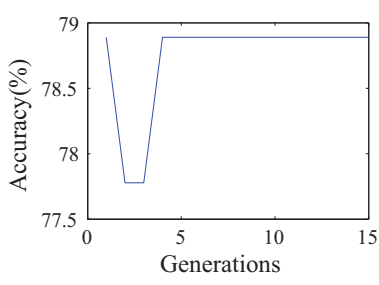

(d) $4 \mathrm{SS}^{3} \mathrm{VM}$

Figure 3 Evolution of the parameters selection. (a), (b), (c), and (d) show the parameters selection procedures of $\mathrm{GSS}^{3} \mathrm{VM}, \mathrm{PSS}^{3} \mathrm{VM}, 3 \mathrm{SS}^{3} \mathrm{VM}$ and $4 \mathrm{SS}^{3} \mathrm{VM}$ respectively (NB: Heart Dataset, labeled proportion 20\%)

In order to compare the classification accuracy between the optimal $\mathrm{SS}^{3} \mathrm{VM}$ models and the non-optimized $\mathrm{SS}^{3} \mathrm{VM}$ models. For each model, 20 groups of parameters are selected randomly and Heart dataset is used to train and test these models. Let $J$ be the index of parameters group. Figure 4 shows the comparison result. It can be easily found that the performance of the optimal $\mathrm{SS}^{3} \mathrm{VM}$ models outperforms that of the models with non-optimized parameters on training set. Though the testing accuracy of the optimal models is not the best, it is good enough. That means the GA/SS ${ }^{3} \mathrm{VM}$ method contributes to the improvement of the classification accuracy, which greatly supports our claims. The performance of the optimal $\mathrm{SS}^{3} \mathrm{VM}$ models on other datasets has the similar results.

Table 4 displays the classification accuracy and CPU time of the optimal $4 \mathrm{SS}^{3} \mathrm{VM}$ under the different labeled proportion. It can be seen that the training and testing accuracy have a little volatility about less than $5 \%$. That means the classification accuracy cannot be remarkably improved when the number of labeled samples increases. It implies that the optimal $4 \mathrm{SS}^{3} \mathrm{VM}$ model has higher computational efficiency, i.e., a small amount labeled samples can be utilized to guarantee the classification accuracy. Thus, the cost of manual labeling can be cut down greatly. 
Table 3 GA/SVM performance on four datasets

\begin{tabular}{llllll}
\hline \multirow{2}{*}{ Data sets } & Models & Optimal & Training & Testing & CPUtime \\
& & parameters & accuracy $\%$ & Testing/\% & /s \\
\hline \multirow{2}{*}{ Heart } & $\mathrm{GSS}^{3} \mathrm{VM}$ & $c=c^{*}=28$ & 77.33 & 74.44 & 0.1814 \\
& $\mathrm{FSS}^{3} \mathrm{VM}$ & $c=c^{*}=7 n=4$ & 77.33 & 73.33 & 2.0603 \\
& $3 \mathrm{SS}^{3} \mathrm{VM}$ & $c=c^{*}=1 k=82$ & 77.77 & 73.33 & 0.1193 \\
& $4 \mathrm{SS}^{3} \mathrm{VM}$ & $c=c^{*}=7 k=2113$ & $\mathbf{7 8 . 8 8}$ & $\mathbf{7 4 . 4 4}$ & $\mathbf{0 . 1 1 4 8}$ \\
\hline \multirow{2}{*}{$\mathrm{QSAR}$} & $\mathrm{GSS}^{3} \mathrm{VM}$ & $c=c^{*}=31$ & 71.55 & 72.15 & 0.4419 \\
& $\mathrm{FSS}^{3} \mathrm{VM}$ & $c=c^{*}=31 n=8$ & $\mathbf{7 2 . 5 4}$ & 74.14 & 2.9183 \\
& $3 \mathrm{SS}^{3} \mathrm{VM}$ & $c=c^{*}=30 k=1564$ & 71.69 & 67.61 & 0.5777 \\
& $4 \mathrm{SS}^{3} \mathrm{VM}$ & $c=c^{*}=28 k=2093$ & 71.97 & $\mathbf{7 4 . 4 4}$ & $\mathbf{0 . 3 5 8 4}$ \\
\hline Wine & $\mathrm{GSS}^{3} \mathrm{VM}$ & $c=c^{*}=25$ & 65.97 & 67.60 & 2.4921 \\
& $\mathrm{FSS}^{3} \mathrm{VM}$ & $c=c^{*}=5 n=3$ & $\mathbf{6 6 . 8 3}$ & 67.60 & 5.3202 \\
& $3 \mathrm{SS}^{3} \mathrm{VM}$ & $c=c^{*}=15 k=1109$ & 65.97 & 67.60 & 5.3202 \\
& $4 \mathrm{SS}^{3} \mathrm{VM}$ & $c=c^{*}=14 k=1294$ & 65.97 & $\mathbf{6 7 . 6 0}$ & $\mathbf{2 . 1 2 9 9}$ \\
\hline Wilt & $\mathrm{GSS}^{3} \mathrm{VM}$ & $c=c^{*}=18$ & 98.54 & 97.78 & 2.6598 \\
& $\mathrm{FSS}^{3} \mathrm{VM}$ & $c=c^{*}=18 n=9$ & 98.54 & 97.78 & 18.9791 \\
& $3 \mathrm{SS}^{3} \mathrm{VM}$ & $c=c^{*}=1 k=100$ & 98.54 & 97.78 & 3.6831 \\
& $4 \mathrm{SS}^{3} \mathrm{VM}$ & $c=c^{*}=28 k=640$ & $\mathbf{9 8 . 5 4}$ & $\mathbf{9 7 . 7 8}$ & $\mathbf{3 . 5 8 7 5}$ \\
\hline
\end{tabular}

Table 4 Effect of percentage of labeled samples to the $4 \mathrm{SS}^{3} \mathrm{VM}$

\begin{tabular}{|c|c|c|c|c|}
\hline Data sets & $\begin{array}{l}\text { Labeled } \\
\text { Proportion }\end{array}$ & $\begin{array}{l}\text { Training } \\
\text { accuracy }\end{array}$ & $\begin{array}{l}\text { Testing } \\
\text { accuracy }\end{array}$ & $\begin{array}{l}\text { CPUtime } \\
/ \mathrm{s}\end{array}$ \\
\hline \multirow[t]{4}{*}{ Heart } & 20 & 78.88 & 74.44 & 0.1148 \\
\hline & 30 & 78.88 & 74.44 & 0.1862 \\
\hline & 40 & 81.66 & 72.22 & 0.2157 \\
\hline & 50 & 82.77 & 74.44 & 0.3143 \\
\hline \multirow[t]{4}{*}{ QSAR } & 20 & 71.69 & 72.44 & 0.3584 \\
\hline & 30 & 72.26 & 73.29 & 0.5167 \\
\hline & 40 & 74.39 & 71.59 & 0.7695 \\
\hline & 50 & 70.98 & 73.01 & 0.7692 \\
\hline \multirow[t]{4}{*}{ Wine } & 20 & 65.97 & 67.60 & 2.4299 \\
\hline & 30 & 66.27 & 66.99 & 3.7138 \\
\hline & 40 & 66.00 & 67.00 & 5.3567 \\
\hline & 50 & 66.03 & 67.66 & 14.7719 \\
\hline \multirow[t]{4}{*}{ Wilt } & 20 & 98.54 & 97.78 & 3.5875 \\
\hline & 30 & 98.54 & 97.78 & 7.2430 \\
\hline & 40 & 98.54 & 97.78 & 7.5340 \\
\hline & 50 & 98.30 & 98.27 & 15.4175 \\
\hline
\end{tabular}




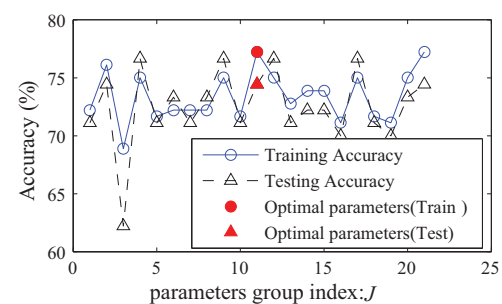

(a) Accuracy of $\mathrm{GSS}^{3} \mathrm{VM}$ with different parameters

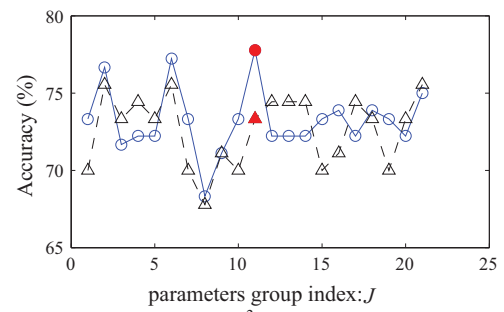

(c) Accuracy of $3 \mathrm{SS}^{3} \mathrm{VM}$ with different parameters

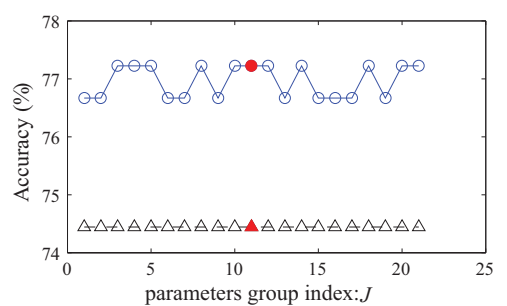

(b) Accuracy of $\mathrm{PSS}^{3} \mathrm{VM}$ with different parameters

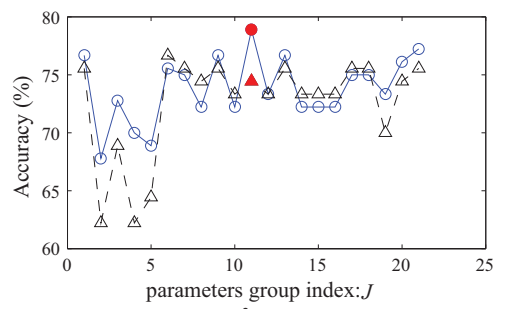

(d) Accuracy of $4 \mathrm{SS}^{3} \mathrm{VM}$ with different parameter

Figure 4 Accuracy comparison between the optimal models and others with arbitrary parameters. (a), (b), (c), and (d) show the compare results of $\mathrm{GSS}^{3} \mathrm{VM}, \mathrm{PSS}^{3} \mathrm{VM}$, $3 \mathrm{SS}^{3} \mathrm{VM}$ and $4 \mathrm{SS}^{3} \mathrm{VM}$ respectively (NB: Heart Dataset, labeled proportion $20 \%$ )

\section{Conclusions}

In this paper, a biquadratic spline function for smoothing the $\mathrm{S}^{3} \mathrm{VM}$ is proposed. According to the analysis about approximation accuracy, the biquadratic spline function has a preferable performance. Further, a new approach named GA $/ \mathrm{SS}^{3} \mathrm{VM}$ method which integrating $\mathrm{SS}^{3} \mathrm{VM}$ and GA is presented. Here, GA is used to optimize parameters of $\mathrm{SS}^{3} \mathrm{VM}$ models. By the hybrid GA/SS${ }^{3} \mathrm{VM}$ method, the optimal $\mathrm{SS}^{3} \mathrm{VM}$ model is obtained. The optimal $\mathrm{SS}^{3} \mathrm{VM}$ model is experimentally evaluated on four real datasets. The results show that the $\mathrm{SS}^{3} \mathrm{VM}$ models optimized by $\mathrm{GA} / \mathrm{SS}^{3} \mathrm{VM}$ approach achieve higher classification accuracy than other non-optimized $\mathrm{SS}^{3} \mathrm{VM}$ models. In particular, the optimal $\mathrm{SS}^{3} \mathrm{VM}$ model based on biquadratic spline function has the desirable classification accuracy and the best computational efficiency. Meanwhile, the classification accuracy of new model is insensitive to the labeled proportion which means that good classification accuracy can be achieved with a small amount labeled samples.

For future work, we intend to apply the kernel function for $\mathrm{SS}^{3} \mathrm{VM}$ and optimize the kernel function, parameters simultaneously.

\section{References}

[1] Vapnik V. The Nature of Statistical Learning Theory. Springer, 1995.

[2] Joachims T. Text categorization with support vector machines: Learning with many relevant features, Springer, 1998.

[3] Melgani F, Bruzzone L. Classification of hyperspectral remote sensing images with support vector machines. IEEE Transactions on Geoscience and Remote Sensting, 2004, 42(8): 1778-1790.

[4] Jonsson K, Kittler J, Li Y P, et al. Support vector machines for face authentication. Image and Vision Computing, 2002, 20(5): 369-375.

[5] Satzger B, Kramer O. Goal distance estimation for automated planning using neural networks and support vector machines. Natural Computing, 2013, 12(1): 87-100. 
[6] Mukkamala S, Janoski G, Sung A. Intrusion detection using neural networks and support vector machines. Proceedings of the 2002 International Joint Conference on Neural Networks, 2002: 1702-1707.

[7] Danenas P, Garsva G. Credit risk evaluation using SVM-based classifier. Business Information Systems 2014 Interbational Workshops, 2010: 7-12.

[8] Shin K S, Lee T S, Kim H J. An application of support vector machines in bankruptcy prediction model. Expert Systems with Applications, 2005, 28(1): 127-135.

[9] Guo Z Q, Wang H Q, Liu Q. Financial time series forecasting using LPP and SVM optimized by PSO. Soft Computing, 2013, 17(5): 805-818.

[10] Fung G, Mangasarian O L. Semi-supervised support vector machines for unlabeled data classifcation. Optimization Methods and Software, 2001, 15(1): 29-44.

[11] Chapelle O, Scholkopf B, Zien A. Semi-Supervised Learning. MIT Press, Cambridge, 2006.

[12] Chapelle O, Zien A. Semi-supervised classification by low density separation. 2004.

[13] Chapelle O, Sindhwani V, Keerthi S. Branch and bound for semi-supervised support vector machines. Conference on Neural Information Processing Systems, 2007: 217-240.

[14] Astorino A, Fuduli A. Nonsmooth optimization techniques for semisupervised classification. IEEE Transactions on Pattern Analysis and Machine Intelligence, 2007, 29(12): 2135-2142.

[15] Reddy I S, Shevade S, Murty M N. A fast quasi-Newton method for semi-supervised SVM. Pattern Recognition, 2011, 44(10): 2305-2313.

[16] Yang L M, Wang L S. A class of smooth semi-supervised SVM by difference of convex functions programming and algorithm. Knowledge-Based Systems, 2013, 41: 1-7.

[17] Lee Y J, Mangasarian O L. SSVM: A smooth support vector machine for classification. Computational Optimization and Applications, 2001, 20(1): 5-22.

[18] Vural V, Fung G, Dy J, et al. Fast semi-supervised SVM classifiers using a priori metric information. Optimization Methods and Software, 2008, 23(4): 521-532.

[19] Liu Y Q, Liu S Y, Gu M T. Polynomial smooth semi-supervised support vector machine. Systems Engineering - Theory \& Practice, 2009, 29(7): 113-118.

[20] Zhang X D, Ma J G. A general cubic spline smooth semi-support vector machine. Chinese Journal of Engineering, 2015, 37(3): 385-389.

[21] Min S H, Lee J, Han I. Hybrid genetic algorithms and support vector machines for bankruptcy prediction. Expert Systems with Applications, 2006, 31(3): 652-660.

[22] Chapelle O, Sindhwani V, Keerthi S S. Optimization techniques for semi-supervised support vector machines. The Journal of Machine Learning Research, 2008, 9: 203-233.

[23] Yuan Y, Huang T. A polynomial smooth support vector machine for classification. Advanced Data Mining and Applications, Springer, 2005.

[24] Dennis J, John E, Moré J J. Quasi-Newton methods, motivation and theory. SIAM Review, 1977, 19(1): 46-89.

[25] Yuan Y X. A modified BFGS algorithm for unconstrained optimization. IMA Journal of Numerical Analysis, 1991, 11(3): 325-332.

[26] Huerta E B, Duval B, Hao J K. A hybrid GA/SVM approach for gene selection and classification of microarray data. Applications of Evolutionary Computing, Springer, 2006.

[27] Zhao X, Huang D, Cheung Y, et al. A novel hybrid GA/SVM system for protein sequences classification. Intelligent Data Engineering and Automated Learning-IDEAL, 2004: 11-16.

[28] Adankon M M, Cheriet M. Genetic algorithm-based training for semi-supervised SVM. Neural Computing and Applications, 2010, 19(8): 1197-1206.

[29] Abdi H, Williams L J. Principal Component Analysis. Wiley Interdisciplinary Reviews: Computational Statistics, 2010, 2(4): 433-459.

[30] Manly B F J. Multivariate statistical methods: A primer. 2nd Edition. Chapman \& Hall, CRC Press, London, 1986.

[31] Tabachnick B G. Fidell L S. Using multivariate statistics. 3rd Edition. 2001.

[32] Noori R, Kerachian R, Darban A, et al. Assessment of importance of water quality monitoring stations using principal component and factor analyses: A case study of the Karoon River. Journal of Water \& Wastewater, 2007, 63(3): 60-69. 